\title{
Reconstruction of Ischial Pressure Sores with Adjacent Muscle Primary Closure and Freestyle Perforator-Based Fasciocutaneous Flaps
}

\author{
Jaehee Kim, Ho Jik Yang \\ Department of Plastic and Reconstructive Surgery, Eulji University College of Medicine, Daejeon, Korea
}

\begin{abstract}
Background: The ischial area is particularly vulnerable to pressure sores, and it is susceptible to recurrent sores even after successful treatment. There are various alternatives to treat ischial pressure sores. In this study, we used adjacent muscle primary closure and freestyle perforator-based fasciocutaneous flap operation with padding of the ischium and obliteration of dead space, considering additional surgery due to recurrence of the surgical site.

Methods: We retrospectively analyzed 10 patients who underwent reconstruction using adjacent muscle primary closure and freestyle perforator-based fasciocutaneous flap to treat ischial pressure sores between March 2014 and February 2017. All operations were performed in two stages. The patients' characteristics, surgical procedure, and clinical course were compared.

Results: The mean age of the patients was 51.7 years (range: 31-68 years), and the mean length of follow-up was 13.3 months (range: 4-20 months). Three patients underwent adjacent muscle primary closure and a posterior thigh fasciocutaneous flap. The rest underwent reconstructive surgery using adjacent muscle primary closure with V-Y advancement fasciocutaneous flap. No recurrences or complications occurred in any of the patients during the follow-up period.

Conclusion: Reconstructive surgery using adjacent muscle primary closure and freestyle perforator-based fasciocutaneous flap is effective for bone padding and dead space obliteration in the treatment of ischial pressure sores. They can also conserve tissue structures and vascularity to allow any additional operations necessitated by recurrent sores.
\end{abstract}

Keywords: Pressure sore, Ischium, Muscle, Perforator flap

\section{Introduction}

Pressure sores are chronic pressure ulcers that can occur due to a patient's general condition, nutrition, position, or movement. They are characterized by skin necrosis resulting from consistent pressure on the skin and muscles, which impedes blood flow [1]. Pressure sores usually occur in weight-bearing bony prominences such as the sacrum, ischial tuberosities, greater trochanters, and calcaneum [2]. In the ischial area, they can occur particularly easily in patients who have undergone surgery and have to maintain a sitting position when eating or moving in a wheelchair [3]. Ischial pressure sores have recurrence rates of $7-48 \%[4,5]$.

The following procedures are used to treat ischial pressure sores: (1) excision of undermined skin, (2) abrasion or resection of the ischial tuberosity, and (3) removal of infected bursae and closure of the defect using a flap [6]. The most important goal of these procedures is to eliminate dead spaces and reduce tension on the skin flap. Various flaps are used in the reconstruction of ischial pressure sores, including the gluteus maximus musculocutaneous flap, inferior gluteal thigh flap, hamstring muscle flap,

\section{Original Article}

Received: March 18, 2018

Revised: August 14, 2018

Accepted: August 17, 2018

\section{Corresponding author:}

Ho Jik Yang

Department of Plastic \& Reconstructive

Surgery, Eulji University Hospital, 95

Dunsanseo-ro, Seo-gu, Daejeon 35233, Korea

Tel: +82-42-611-3029

Fax: +82-42-259-1111

E-mail: drhjyang@eulji.ac.kr

This is an Open Access article distributed under the terms of the Creative Commons Attribution Non-Commercial License (http://creativecommons.org/licenses/by-nc/4.0/) which permits unrestricted non-commercial use, distribution, and reproduction in any medium, provided the original work is properly cited.

C) 2018 Korean Wound Management Society 
biceps femoris muscle flap, tensor fascia lata flap, and gracilis muscle flap [7].

Since Koshima et al. introduced the perforator flap [8], the technique has been favored as it can conserve the underlying muscles, reduce donor site morbidity, and improve esthetic outcome. Specifically, the superior gluteal artery perforator (SGAP) flaps and the inferior gluteal artery perforator (IGAP) flaps are the most widely used in the treatment of ischial sores $[7,9]$. The use of muscle flaps to treat pressure sores allows for dead space obliteration, pressure distribution, and reliable blood supply, and thus has advantages in terms of infection control [10]. The fasciocutaneous flap lacks volume and can only obliterate dead spaces to a limited extent. In contrast, the musculocutaneous flap has sufficient volume and blood flow, and can be a useful treatment option for deeply infected wounds such as pressure sores [11].

Consistent application of pressure to the ischial area in daily life can induce recurrent sores, and many patients with ischial sores undergo several operations during their lifetime. However, because the gluteal region contains limited vascular pedicles, only a limited number of operations can be performed in this region. Therefore, when selecting flaps, surgeons must take into account the possibility that sores may recur in the future [7].

In the present study, adjacent muscle primary closure was performed to obliterate dead space and sufficiently cover of the ischial bone. And a fasciocutaneous flap was followed that can reduce skin tension and increase flap survival. This dualflap technique lowered donor site morbidity and reconstructed recurrent ischial pressure sores more effectively than existing methods that use the gracilis or biceps muscle flap.

\section{Methods}

The present study included 10 patients who had developed Grade IV pressure sores in the ischial area between March 2014 and February 2017. When operating on pressure sores, infection control is as important as surgical technique. Also, wound closure should be performed as soon as possible after admission to reduce hospital infection possibility with appropriate selection of antibiotics for infection control. Wound closure was performed at an average of 7 days after admission, as soon as bacterial culture and antimicrobial susceptibility test results were obtained.

All patients underwent debridement, including ischial bone pressure point ostectomy, and negative pressure wound therapy
(NPWT) in a first-stage operation. As they awaited their wound culture results, they were subjected to infection control with broad-spectrum antibiotics. Seven days after the first-stage operation, the patients underwent a second-stage operation involving adjacent muscle primary closure and freestyle perforator-based fasciocutaneous flap operation with proper antibiotics use. Twenty-one days after surgery, computed tomography (CT) was performed to look for evidence of complications. Defect sizes, surgical methods, complications, and recurrence rates were compared using the patients' medical records.

\section{Surgical technique}

All patients underwent a two-stage operation following admission to the hospital. In the first stage, the patient was placed on an operating table in the prone position with the hip flexed. A wound culture was performed to ensure the appropriate antibiotics were chosen, and debridement was performed. All non-viable, fibrous, and infected tissues were radically excised, and a bursectomy was performed. To ensure a smooth contour was created at the ischial pressure points, both an ostectomy and rasping were performed. A bone culture was performed on lesions with suspected osteomyelitis, and NPWT was applied to control infection. The pressure of the vacuum pump was set to $100 \mathrm{mmHg}$ in a continuous mode for exudate and bleeding absorption. NPWT was changed every 72 hours while waiting for wound culture and bone culture results.

The wound culture results were obtained an average of 7 days later, and the second stage of the operation was performed at this time. Specifically, the patient was placed on an operating table in the prone position with the hip flexed, and meticulous debridement and rongeuring of the ischial bony prominence were performed. A portable Doppler was used to look for a reliable perforator around the defect area (Fig. 1). To fill the dead space and cover the exposed ischium, a muscle flap constructed from adjacent muscles around defect area such as gluteus maximus, adductor magnus, biceps femoris or semitendinosus was used. Mainly used muscle was gluteus maximus, and adductor magnus, biceps femoris or semitendinosus could be used when the defect area was located in medial and caudal side of ischial tuberosity. To minimize muscle flap tension, the muscle flaps were elevated on both sides of the long axis of the defect area created after debridement. Using subfascial dissection, adjacent muscle around defect area was randomly selected and dissected at periphery of the defect area, while carefully checking the muscle tissue layer and 


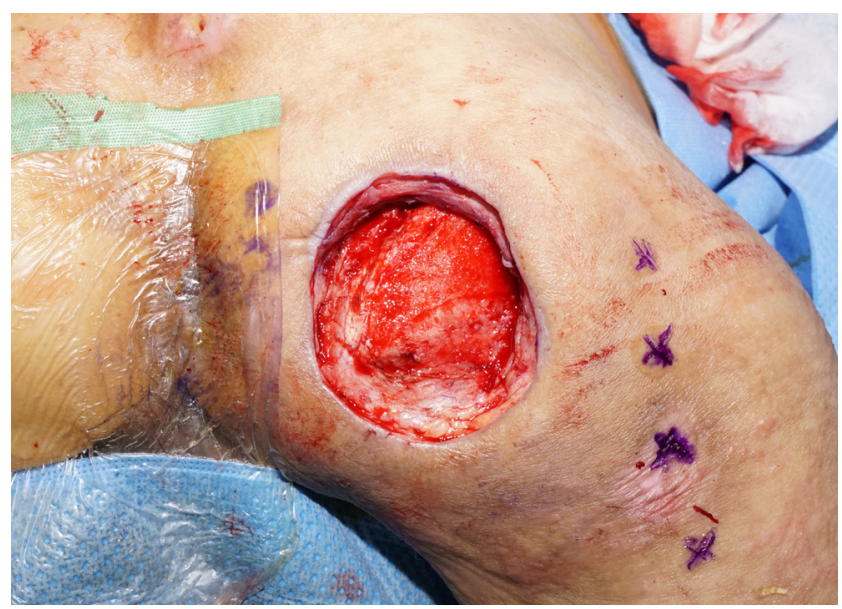

Fig. 1. Periwound perforator mapping. Reliable perforators were found around the defect area by Doppler.

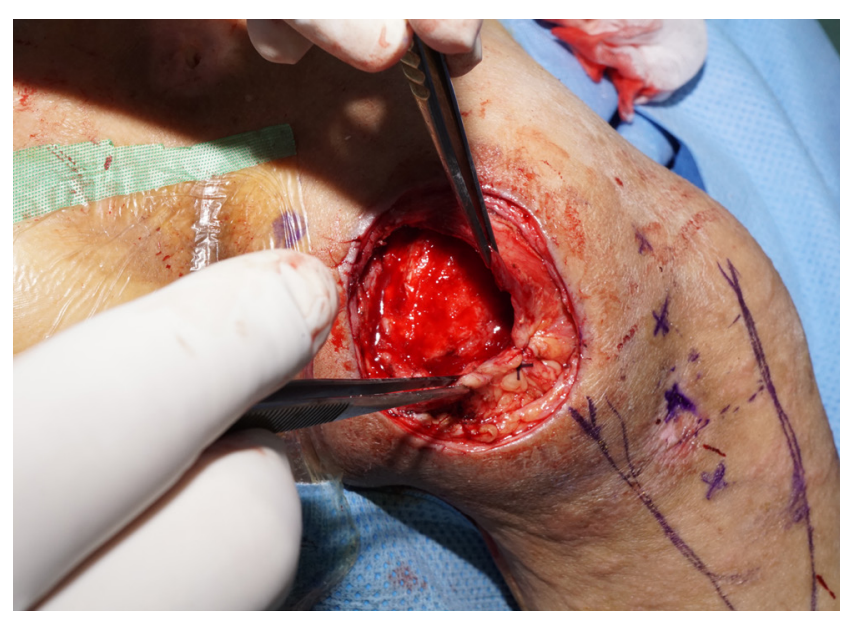

Fig. 2. Primary closure with adjacent muscle. Adjacent muscle primary closure was performed after the possibility of primary closing was confirmed by manual advancement of undermined muscles.

avoiding the perforator along the margin of the defect area. Then supraperiosteal dissection was performed to mobilize the adjacent muscle flaps, using electrocautery. While mobilizing both sides of the muscle layer, we confirmed the possibility of primary muscle closing by manual advancement of mobilized muscle flaps. An adjacent muscle primary closure was then performed with Vicryl 1-0 simple interrupted suture, ensuring that the exposed bony surface was sufficiently covered by the sutured muscle flaps on both sides (Fig. 2). Next, extended subfascial dissection was performed to create and elevate a freestyle fasciocutaneous flap with the perforator as the base (Fig. 3). The flap was moved to cover the defect without causing any tension, and the donor site was closed primarily

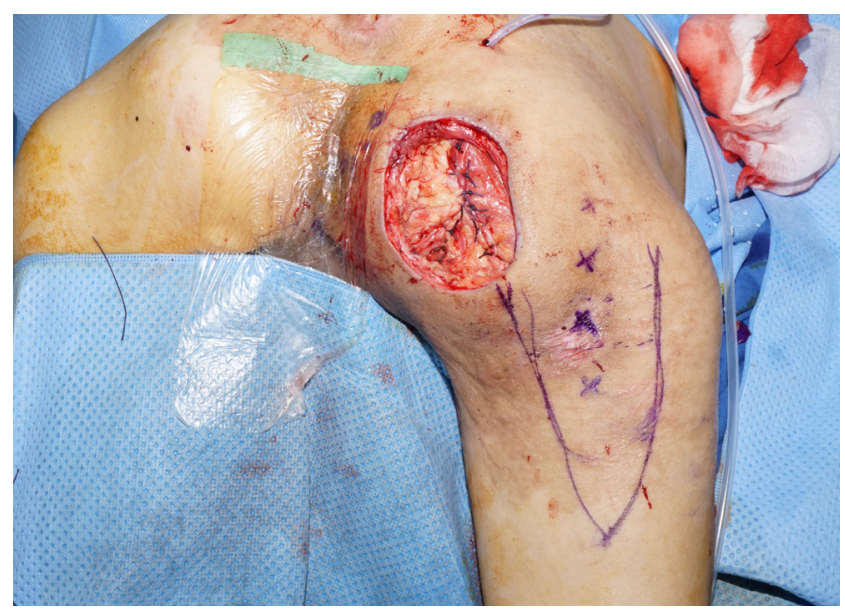

Fig. 3. Posterior thigh perforator flap design. Adjacent muscle primary closure was performed after the possibility of primary closing was confirmed by manual advancement of undermined muscles.

with minimal subcutaneous undermining.

The mean duration of the first stage was 1 hour, while that of the second stage was 2 hours. Two negative suction drainage catheters were used on the second stage operation, and they were removed once the drainage amount reached below 10 $\mathrm{mL}$ at least 7 days after surgery. All patients maintained the prone position for 3 weeks after surgery.

\section{Results}

Of the 10 patients, seven were men, and three were women. The patients' age ranged from 31 to 68 years; their mean age was 51.7 years. One patient was quadriplegic due to Sandhoff disease, and the remaining patients had paraplegia due to spinal cord injury. Three of the patients underwent adjacent muscle primary closure and a posterior thigh fasciocutaneous flap. The others underwent reconstructive surgery using adjacent muscle primary closure and a V-Y advancement fasciocutaneous flap. Post-operative CT performed 21 days after surgery showed sufficient coverage of the ischial bone, and there was no fluid collection in any of the patients. No shortterm complications occurred. One patient (10\%) had post-operative wound dehiscence 3 months after surgery, but the wound was secondarily healed. The mean duration of followup was 13.3 months (range: 4-20 months), and no recurrences have been observed to date.

\section{Case 1}

A 31-year-old woman with Sandhoff disease was diagnosed 

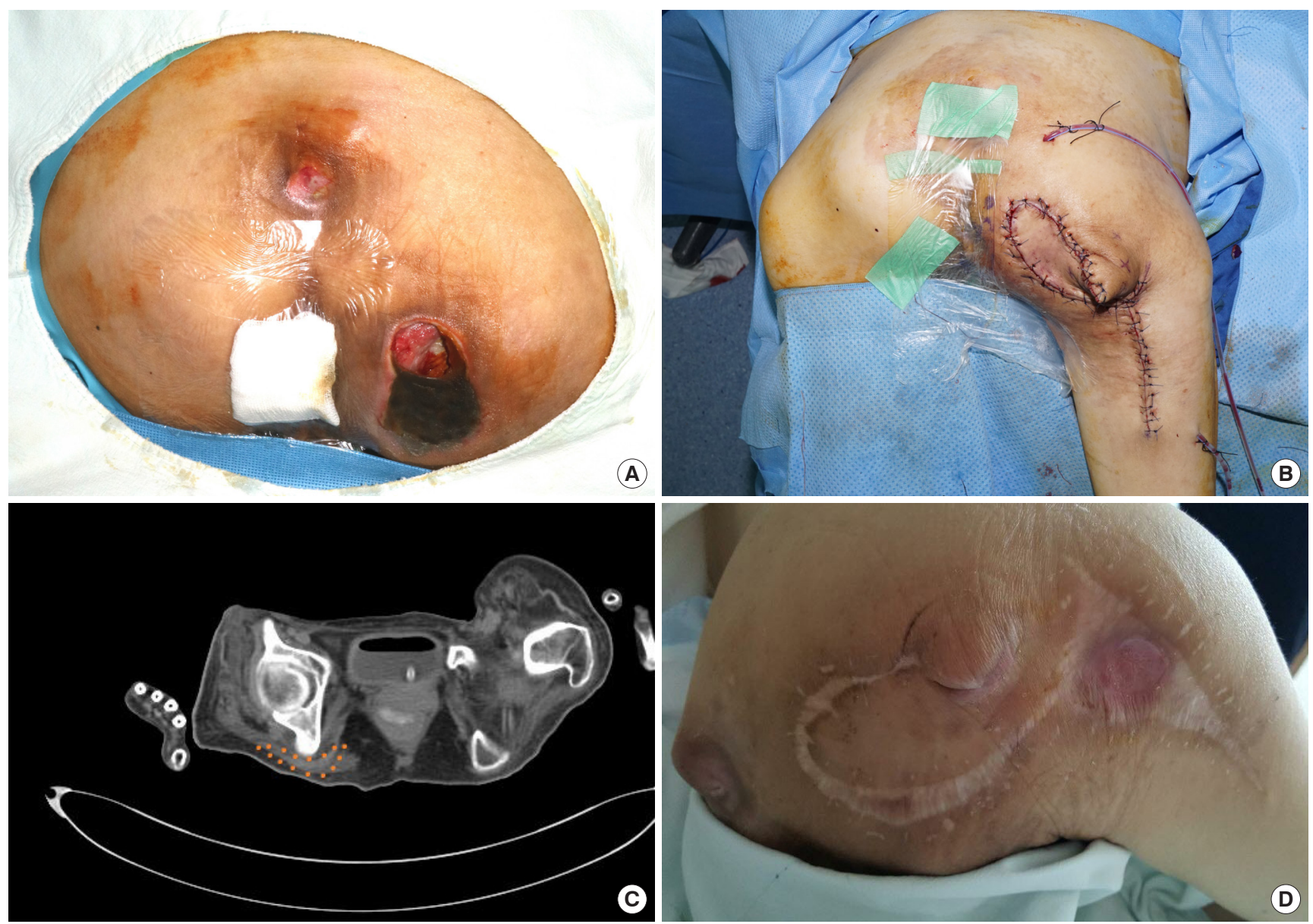

Fig. 4. A case of right ischial pressure sore (Case 1). (A) A dead space of about $10 \mathrm{~cm} \times 9 \mathrm{~cm}$ in size was observed in the $3 \mathrm{~cm} \times 3 \mathrm{~cm}$ skin opening. (B) Defect area was sufficiently closed using adjacent muscle primary closure and a posterior thigh fasciocutaneous flap. (C) Postoperative day 21 CT scan. The dotted area shows that the ischial pressure point was sufficiently padded by the adjacent muscle primary closure. (D) Postoperative month 20 photo. No additional pressure ulcers occurred and wound is managed by home-care service.

with a right ischial pressure sore and referred to the department of plastic and reconstructive surgery. The initial skin defect size was approximately $3 \mathrm{~cm} \times 3 \mathrm{~cm}$, and the size of the dead space was about $10 \mathrm{~cm} \times 9 \mathrm{~cm}$ (Fig. 4A). Debridement and ischial bone ostectomy were performed as the first stage of surgery, followed by NPWT for 1 week. Wound and bone culture results confirmed the presence of Pseudomonas aerugino$s a$ before the second stage of surgery, and ciprofloxacin was administered for 6 weeks, accordingly. The second stage of the operation involved adjacent muscle primary closure and reconstruction using a posterior thigh fasciocutaneous flap (Fig. 4B). No post-operative complications occurred. Post-operative CT showed that the ischial bone was sufficiently padded by muscles, and no fluid collection was observed (Fig. 4C). The pressure sore did not recur during the 20-month post-operative follow-up (Fig. 4D).

\section{Case 2}

A 65-year-old man had become paraplegic due to an injury of the third thoracic vertebra after surgery for an aortic aneurysm. He was diagnosed with a right ischial pressure sore and admitted to the present institution after undergoing primary incision and drainage. The initial skin defect was approximately $10 \mathrm{~cm} \times 5 \mathrm{~cm}$ in size, while the dead space measured about $14 \mathrm{~cm} \times 8 \mathrm{~cm}$. An initial wound culture confirmed the presence of methicillin-resistant Staphylococcus aureus (MRSA). This result was corroborated after the first operation, and vancomycin was administered accordingly. During the second operation, adjacent muscle primary closure and reconstruction using a V-Y advancement fasciocutaneous flap were performed following rongeuring of the ischial bony prominence (Fig. 5). No post-operative complications occurred, and no fluid collection was observed on post-operative CT scan. Al- 

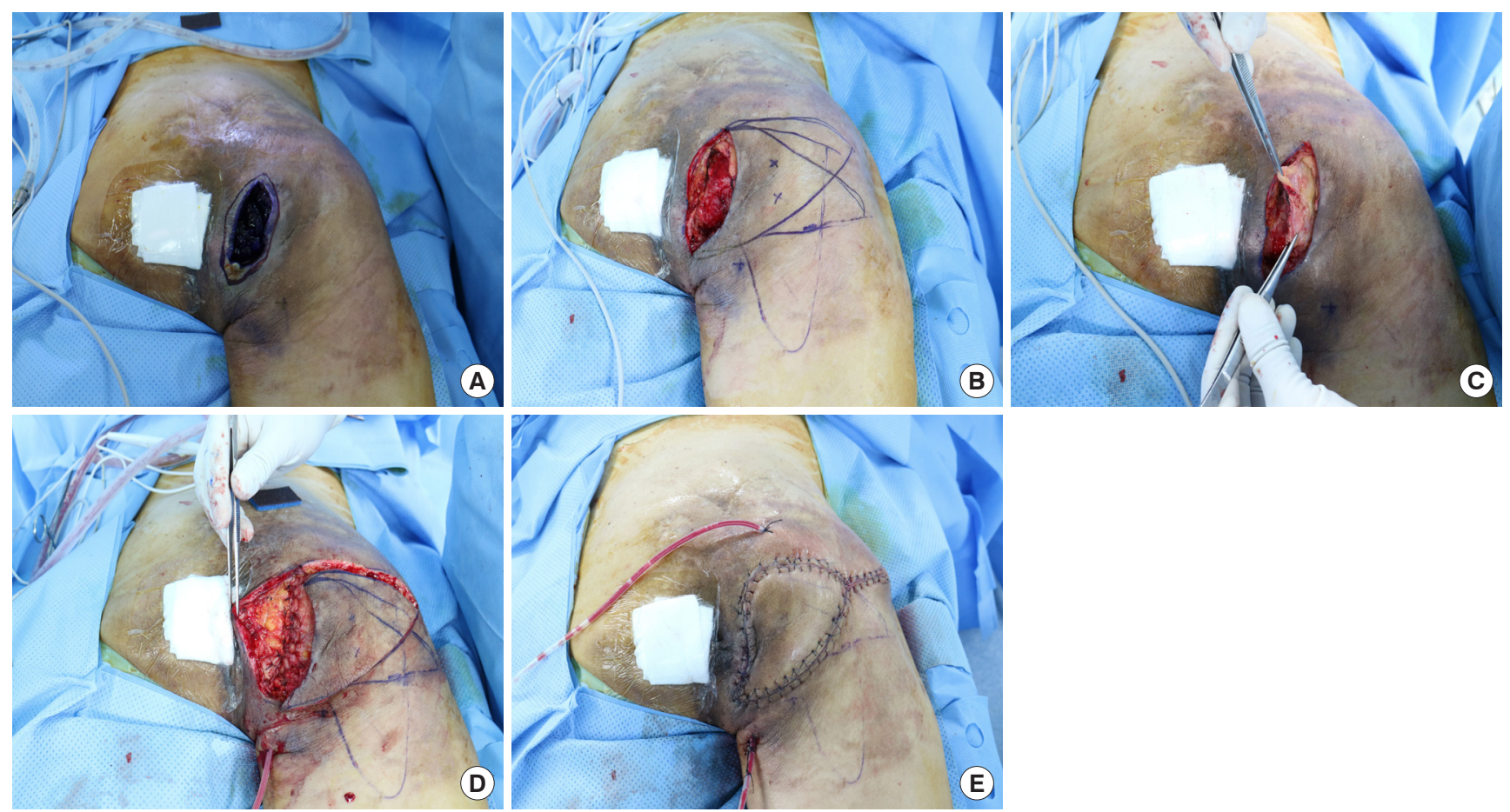

Fig. 5. Second stage operation procedure (Case 2). (A) The second-stage pre-operative photo. After NPWT application, the secondstage operation was performed 6 days later. (B) Reliable perforators were found around the defect area by Doppler. (C) An adjacent muscle primary closure was performed for bony prominence padding. (D) The V-Y advancement fasciocutaneous flap was elevated following adjacent muscle primary closure. (E) Defect area was sufficiently closed using adjacent muscle primary closure and a $V$ - $Y$ advancement fasciocutaneous flap.

though dehiscence occurred at the triangular point of the flap, it was secondarily healed through dressing. The pressure sore did not recur during the 12-month post-operative follow-up.

\section{Discussion}

Consistent application of pressure to the ischial region during daily life can induce ischial pressure sores, even after successful flap closure. Many patients require multiple flap surgeries as pressure sores repeatedly form in the ischial, trochanteric, and sacral areas during their lifetime. It is important that these operations be planned so that tissue structures and vascularity are conserved in case additional operations are needed in the future [12].

Several alternatives are available for the surgical treatment of ischial pressure sores, including gluteus maximus musculocutaneous flap, inferior gluteal thigh flap, hamstring muscle flap, biceps femoris muscle flap, tensor fascia lata flap, and gracilis muscle flap [7]. Ideally, flaps for ischial pressure coverage must be well-vascularized and have sufficient bulkiness to obliterate the dead space. They must also conserve potential vascular pedicles for any future reconstruction [13]. In patients with a reduced sensation in the limbs, whatever the cause, consistent position changes are required to prevent other sites from being affected by pressure sores. Flexion and extension of the lower extremities during these position changes apply a large amount of tension to the ischial area. Therefore, to minimize the amount of tension applied to the surgical site following treatment, surgeons should select flaps that are not too bulky and that cause minimal damage to the normal anatomy of the lower extremities.

Muscle flaps are the best option for dead space obliteration, and the gracilis muscle in particular is widely used in the reconstruction of ischial pressure sores [14]. However, in the present study, instead of muscle flap elevation using the gracilis or biceps muscle flap, the ischium was padded using adjacent muscle primary closure around the defect area, which uses a local muscle flap, and the dead space was obliterated using a well-vascularized tissue. This method does not require any skin incision to be made during the process of muscle flap elevation, and it does not damage deep tissue structures. Therefore, donor site morbidity and surgery duration are re- 
duced. Volume was added to the skin defect area through using a fasciocutaneous flap, and this dual padding disperses pressure and reduced tension to increase flap survival. Since adjacent muscle primary closure does not involve excessive muscle flap elevation, the perforators around the defect are well-conserved. Therefore, the technique allows for a wide range of choices in terms of fasciocutaneous flap design.

In the present study, a hand-held Doppler was used to secure reliable perforators, and either a posterior thigh fasciocutaneous flap or $\mathrm{V}-\mathrm{Y}$ advancement fasciocutaneous flap was used to perform a minimal-tension flap closure. All secondstage operations were finished within 2 hours. During the mean follow-up period of 13.3 months, no serious complications occurred, aside from one case of wound dehiscence that occurred 3 months after surgery. No fluid collection was observed on CT scans obtained from all patients 21 days after surgery. In all patients, the ischial bony prominence was properly padded with muscle tissues on CT scans.

The inferior gluteus maximus myocutaneous flap is the most widely used in the treatment of ischial pressure sores, and it has shown excellent outcomes $[7,15]$. However, in the present study, the defects were successfully closed using the dual flap technique, which uses both adjacent muscle primary closure and a fasciocutaneous flap of adequate bulkiness, and which involves bone padding and dead space obliteration. Patients who undergo surgery with this technique receive little damage to tissue structures and have relatively well-conserved pedicles. Therefore, surgeons have a wider range of tissue to choose from to create new flaps if the patients develop recurrent sores on the defect area.

The major drawbacks limiting the range of application in performing this procedure include numerous prior operations and severe muscle atrophy in long-term paralysis. However, in patients with moderate muscle atrophy, a dual padding technique using thin-walled muscle padding and a fasciocutaneous flap may be applied to allow sufficient padding.

To reconstruct pressure sores in the ischial area, bone padding and dead space obliteration are crucial. Surgical techniques using both adjacent muscle primary closure and a freestyle perforator-based fasciocutaneous flap are relatively simple, retain the benefits of muscle flaps, and can conserve tissue structures and vascularity for any future operations necessitated by recurrent sores.

\section{References}

1. Bass MJ, Philips LG. Pressure sores. Curr Probl Surg 2007; 44:101-43.

2. Hunter IA, Saker R. Managing pressure sores. Surgery 2011;29:518-22.

3. Lee SS, Huang SH, Chen MC, et al. Management of recurrent ischial pressure sore with gracilis muscle flap and V-Y profunda femoris artery perforator-based flap. J Plast Reconstr Aesthet Surg 2009;62:1339-46.

4. Hentz VR. Management of pressure sores in a specialty center. A reappraisal. Plast Reconstr Surg 1979;64:683-91.

5. Tavakoli K, Rutkowski S, Cope C, et al. Recurrence rates of ischial sores in para- and tetraplegics treated with hamstring flaps: an 8-year study.Br J Plast Surg 1999;52:476-9.

6. Vasconez LO, Schneider WJ, Jurkiewicz MJ. Pressure sores. Curr Probl Surg 1977;50:374-9.

7. Foster RD, Anthony JP, Mathes SL, et al. Ischial pressure sore coverage: a rationale for flap selection. Br J Plast Surg 1997;50:374-9.

8. Koshima I, Moriguchi T, Soeda S, et al. The gluteal perforator-based flap for repair of sacral pressure sores. Plast Reconstr Surg 1993;91:678-83.

9. Coskunfirat OK, Ozgentas HE. Gluteal perforator flaps for coverage of pressure sores at various locations. Plast Reconstr Surg 2004;113:2012-7.

10. Thiessen FE, Andrades P, Blondeel PN, et al. Flap surgery for pressure sores: should the underlying muscle be transferred or not? J Plast Reconstr Aesthet Surg 2011;64:84-90.

11. Cushing CA, Phillips LG. Evidence-based medicine: pressure sores. Plast Reconstr Surg 2013;132:1720-32.

12. Lin PY, Kuo YR, Tsai YT. A reusable perforator-preserving gluteal artery-based rotation fasciocutaneous flap for pressure sore reconstruction. Microsurgery 2012;32:189-95.

13. Rajacic N, Gang RK, Dashiti H, et al. Treatment of ischial pressure sores with an inferior gluteus maximus musculocutaneous island flap: an analysis of 31 flaps. Br J Plast Surg 1994;47:431-4.

14. Labandter HP. The gracilis muscle flap and musculocutaneous flap in the repair of perineal and ischial defects. $\mathrm{Br} \mathrm{J}$ Plast Surg 1980;33:95-8.

15. Sameem M, Au M, Wood T, et al. A systematic review of complication and recurrence rates of musculocutaneous, fasciocutaneous, and perforator-based flaps for treatment of pressure sores. Plast Reconstr Surg 2012;130:67e-77e. 